\title{
The most beautiful COPD chart in the world: all together to end COPD!
}

To the Editor:

Since its inception in 2002, World COPD Day is celebrated to raise awareness and reduce COPD-related burden. In spite of this global effort, COPD remains poorly recognised. Indeed, Bовнм et al. [1], using Google Trends, noted the rather limited global awareness of COPD, which rates eighth within the top 10 causes of disease burden searched. On a positive note, they identified a seasonal trend, with a peak activity in November, that could be related to the increased incidence of acute exacerbations during the winter period, but it may also reflect the "noise" created by initiatives like the COPD Awareness Month and World COPD Day. The 2019 theme for World COPD Day is “All Together to End COPD”. Optimistically, recent evidence of projected global COPD mortality rates indicates this theme to actually be more than wishful thinking.

Last month, Simon Hay, Professor of Global Health at the Institute for Health Metrics and Evaluation, at the University of Washington in Seattle, published the self-coined "most beautiful chart in the world" [2], depicted from the Global Burden of Disease (GBD) Study in 2017, with under-5 mortality rates per 10000 children in 103 low- and middle-income countries expected to be more than halved from 1970 to 2030. This is good news.

We previously showed a downward trend in COPD mortality rates from 1994 to 2010 in the 27 countries of the European Union [3], that could serve as a reference to allow informed policy making. Using the most advanced prognosticating models, we explored COPD deaths and age-standardised mortality rates with GBD Foresight from 1990 to 2016, and their projections up to 2040 [4]. Figure 1a illustrates the crude COPD mortality rates per 100000 population by GBD region by calendar year. Most world regions experience smaller numbers than Asia, where actually deaths in East Asia peaked in 1994 with 1.4 million, intersected with South Asia from 2013 to 2023, and are projected to peak again up to 2040 with nearly 1.6 million COPD deaths, mainly due to demographic changes, such as population growth and global ageing. Therefore, age-standardisation must be applied, as it is considered the most valid method when comparing rates from different time periods, eliminating the effect of differences in population age structures, such as population growth and ageing [5]. Therefore, in figure $1 \mathrm{~b}$ the all-ages age-standardised COPD mortality rates are depicted, and indeed it might be coined the most beautiful COPD chart in the world. Only East Asia, South Asia and Oceania will have discernible mortality rates in females and males by 2040, while all other world regions will have rates well below 30 deaths per 100000 per year. By pooling all available evidence, GBD's Spatiotemporal Gaussian process meta-regression methods appear valid and reliable [6], so we should not be considered naïve if we dream of a world where tobacco control measures, paired with reductions in environmental pollution in cities, electric cars, cleaner working environments, better treatment of respiratory and non-respiratory comorbidities [7], and reductions of universally high COPD underdiagnosis [8], will sharply reduce the COPD burden [9]. Hurdles include old and new forms of smoking, such as marijuana, e-cigarettes and other tobacco "heat-not-burn" delivery modes. However, it is possible for all to be controlled and reduced to a minimum with law, medical and societal efforts.

Once again, the only constant element in COPD is change [10]. Fortunately, it seems as our war against this scourge is starting to be won [11]. We'll meet again in 2040 to confirm if these "beautiful" COPD mortality trends are close to zero, and that the many millions of COPD patients will die with COPD, but not from COPD [12]. So, happy birthday COPD! And let us aim to end COPD not only today, but every day. 


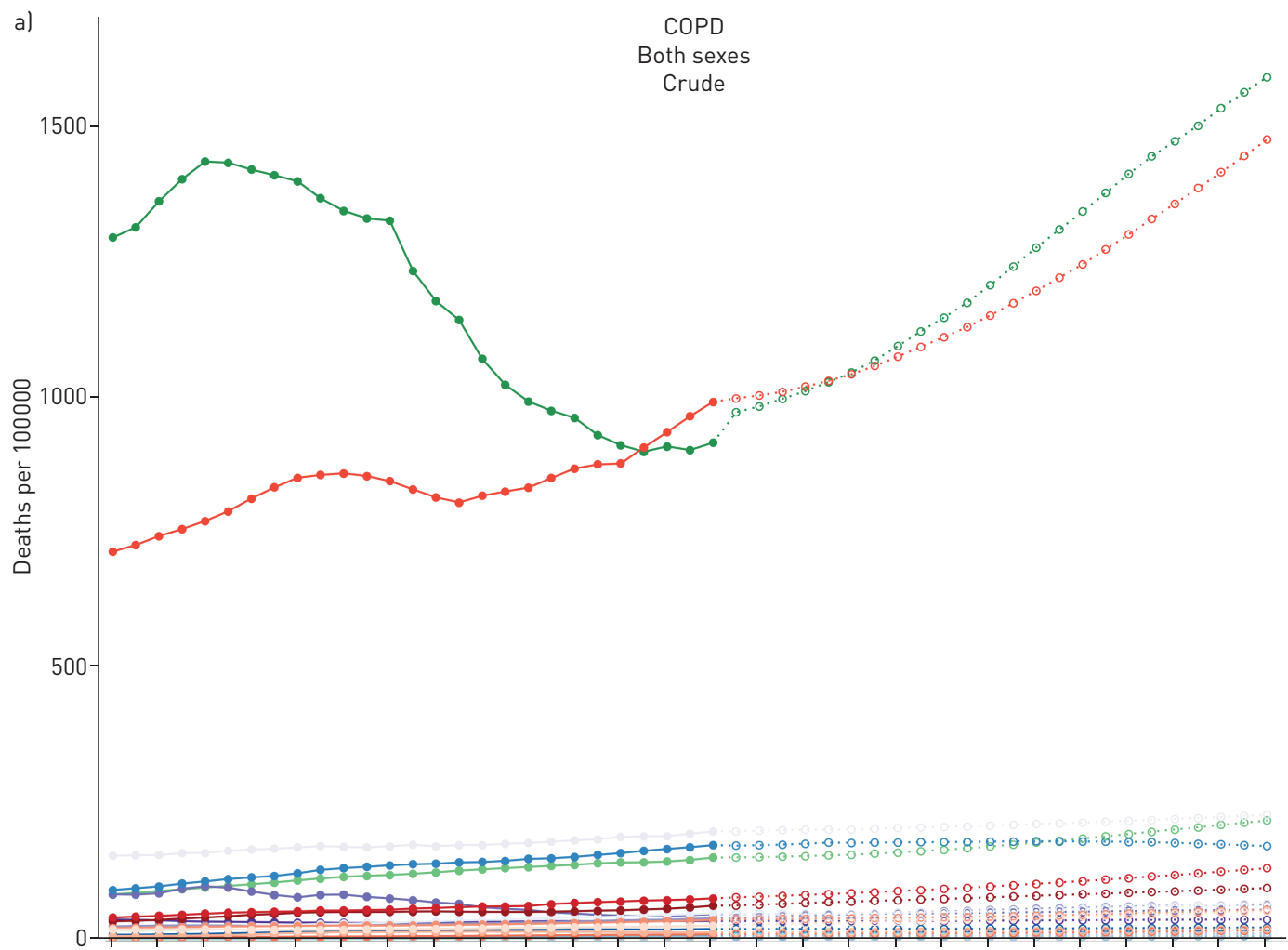

- East Asia

$\rightarrow$ Southeast Asia

$\rightarrow$ Oceania

Central Asia

$\rightarrow$ Central Europe

$\rightarrow$ Eastern Europe

$\rightarrow$ High-income Asia Pacific

$\rightarrow$ Australasia

Western Europe

$\rightarrow$ Southern Latin America

$\rightarrow$ High-income North America

$\rightarrow$ Caribbean

$\rightarrow$ Andean Latin America Central Latin America

$\rightarrow$ Tropical Latin America

$\rightarrow$ North Africa and Middle East

$\rightarrow$ South Asia

- Central Sub-Saharan Africa

$\rightarrow$ Eastern Sub-Saharan Africa

- Southern Sub-Saharan Africa

- Western Sub-Saharan Africa

- 1990-2016

‥ 2017-2040 b)

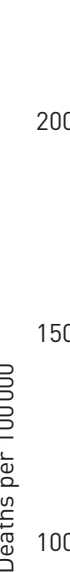
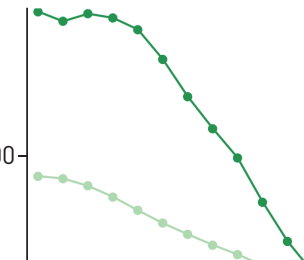

50
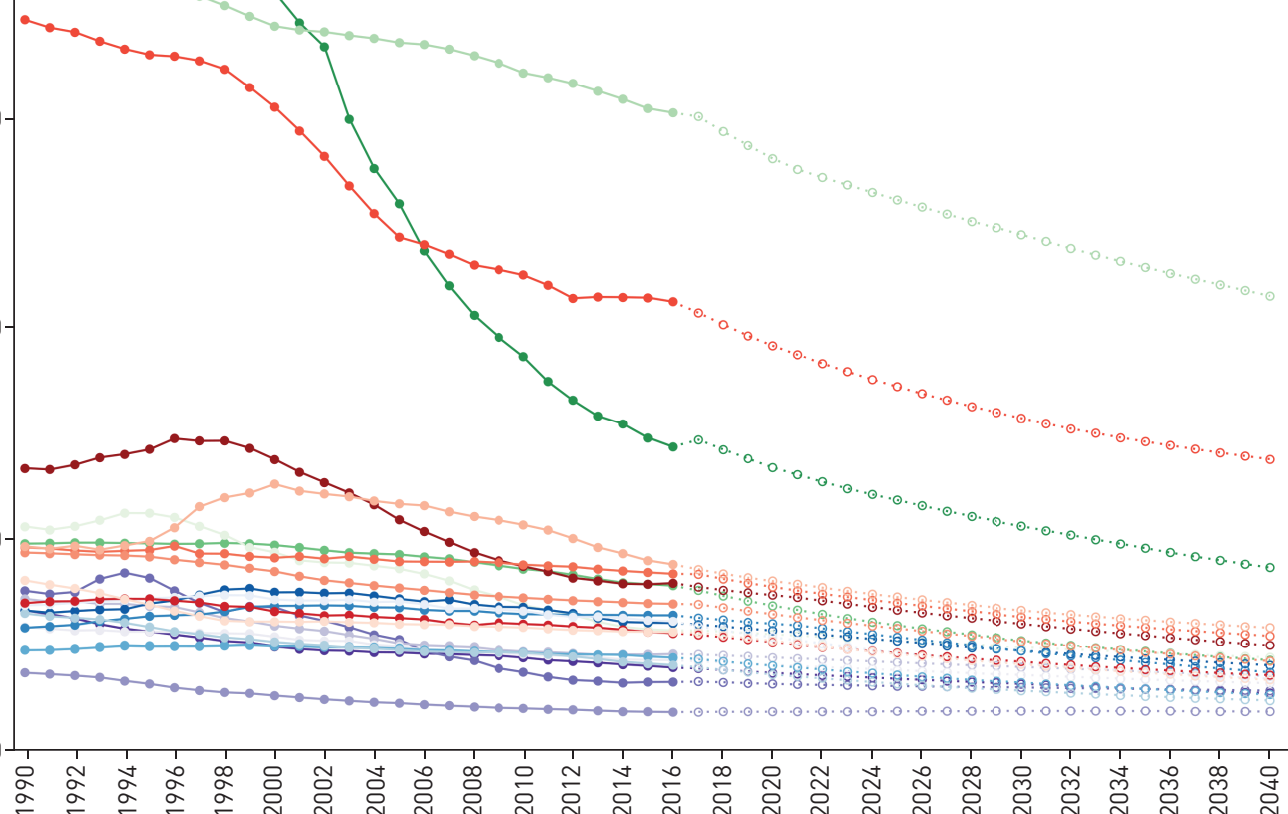

Both sexes

Age standardised

Year $\rightarrow$ East Asia

$\rightarrow$ Southeast Asia

$\rightarrow$ Oceania

Central Asia

- Central Europe

$\rightarrow$ Eastern Europe

$\rightarrow$ High-income Asia Pacific

- Australasia Western Europe

$\rightarrow$ Southern Latin America

$\rightarrow$ High-income North America

$\rightarrow$ Caribbean

- Andean Latin America Central Latin America

- Tropical Latin America

$\rightarrow$ North Africa and Middle East

$\rightarrow$ South Asia

- Central Sub-Saharan Africa

$\rightarrow$ Eastern Sub-Saharan Africa

$\rightarrow$ Southern Sub-Saharan Africa

- Western Sub-Saharan Africa

- 1990-2016

… 2017-2040

FIGURE 1 COPD mortality rates per 100 000, all ages, both sexes, from 1990 to 2016, and the projection until the year 2040: al crude; b) agestandardised. 
Joan B. Soriano $\oplus^{1,2}$, Julio Ancochea ${ }^{1,2}$ and Bartolomé R. Celli $\odot^{3}$

${ }^{1}$ Servicio de Neumología, Hospital Universitario de la Princesa, Madrid, Spain. ${ }^{2}$ Centro de Investigación en Red de Enfermedades Respiratorias (CIBERES), Instituto de Salud Carlos III (ISCIII), Madrid, Spain. ${ }^{3}$ Pulmonary and Critical Care Division, Brigham and Women's Hospital, and Harvard Medical School, Boston, MA, USA.

Correspondence: Joan B. Soriano, Hospital Universitario de la Princesa, Diego de León 62, Madrid, 28030, Spain. E-mail: jbsoriano2@gmail.com

Received: 17 Oct 2019 | Accepted after revision: 23 Oct 2019

Conflict of interest: J.B. Soriano participated in speaking activities, advisory committees and consultancies during the period 2015-2019 sponsored by: Almirall, AstraZeneca, Boehringer Ingelheim, CHEST, Chiesi, ERS, GEBRO, Grifols, GSK, Linde, Lipopharma, Mundipharma, Novartis, Pfizer, RiRL, Rovi, Sandoz, SEPAR and Takeda; and declares not receiving ever, directly or indirectly, funding from the tobacco industry or its affiliates. Julio Ancochea has received honoraria from Air Liquide, Almirall, AstraZeneca, Boehringer Ingelheim, Chiesi, Esteve, Ferrer, GSK, Linde Healthcare, Menarini, Mundipharma, Novartis, Roche, Rovi,Sandoz and Teva during the period 2014-2019 for speaking at conferences, moderatorships, scientific consultancy services and participating in clinical trials. B.R. Celli reports grants and other (research site) from AstraZeneca, personal fees for consultancy and committee work from GlaxoSmithKline, personal fees for consultancy from Boehringer Ingelheim, Sanofi-Aventis, Menarini, Chiesi and Pulmonx, outside the submitted work.

\section{References}

1 Boehm A, Pizzini A, Sonnweber T, et al. Assessing global COPD awareness with Google Trends. Eur Respir J 2019; 53: 1900351

2 Hay SI. Maintaining progress for the most beautiful chart in the world. Int Health 2019; 11: 344-348.

3 López-Campos JL, Ruiz-Ramos M, Soriano JB. Mortality trends in chronic obstructive pulmonary disease in Europe, 1994-2010: a joinpoint regression analysis. Lancet Respir Med 2014; 2: 54-62.

4 Institute for Health Metrics and Evaluation (IHME). GBD Foresight. Seattle, IHME, University of Washington, 2015http://ihmeuw.org/4xre. Date last accessed: October 13, 2019.

5 Abramson JH, Abramson ZH. Making Sense of Data: A Self-Instruction Manual on the Interpretation of Epidemiological Data. Oxford and New York, Oxford University Press, 2001.

6 Institute for Health Metrics and Evaluation (IHME). GBD Compare. Seattle, IHME, University of Washington, 2015. http://vizhub.healthdata.org/gbd-compare. Date last accessed: October 13, 2019.

7 Celli BR, Wedzicha JA. Update on clinical aspects of chronic obstructive pulmonary disease. N Engl J Med 2019; 381: $1257-1266$.

8 Soriano JB, Zielinski J, Price D. Screening for and early detection of chronic obstructive pulmonary disease. Lancet 2009; 374: 721-732.

9 Polverino F, Celli B. The challenge of controlling the COPD epidemic: unmet needs. Am J Med 2018; 131: 1-6.

10 Soriano JB. The evolution of COPD species; or, something is changing for good in COPD. Eur Respir J 2019; 53: 1900762.

11 Sin DD, King GG. COPD: lessons learned, forging a fantastic future. Respirology 2017; 22: 632-633.

12 Hansell AL, Walk JA, Soriano JB. What do chronic obstructive pulmonary disease patients die from? A multiple cause coding analysis. Eur Respir J 2003; 22: 809-814. 